\title{
Homotopy Analysis Method for Large-Amplitude Free Vibrations of Strongly Nonlinear Generalized Duffing Oscillators
}

\author{
Youhua Qian, Dongxu Ren, Shengmin Chen*, Lin Ping \\ College of Mathematics, Physics and Information Engineering, Zhejiang \\ Normal University, Jinhua, China



Received June 30, 2012; revised August 2, 2012; accepted August 16, 2012

\begin{abstract}
In this study, the homotopy analysis method (HAM) is used to solve the generalized Duffing equation. Both the frequencies and periodic solutions of the nonlinear Duffing equation can be explicitly and analytically formulated. Accuracy and validity of the proposed techniques are then verified by comparing the numerical results obtained based on the HAM and numerical integration method. Numerical simulations are extended for even very strong nonlinearities and very good correlations which achieved between the results. Besides, the optimal HAM approach is introduced to accelerate the convergence of solutions.
\end{abstract}

Keywords: Strongly Nonlinear Vibration; Generalized Duffing Equation; Homotopy Analysis Method

\section{Introduction}

Nonlinear Duffing equation is a simple mathematical model which describes the resonance and chaotic phenomenon. In science and engineering many nonlinear vibration problems can be transformed into the Duffing equation to research [1]. From a certain point, the real significance of the research on nonlinear Duffing system attracts a lot of scholars and several ingenious analytical methods have been developed for dealing with the nonlinear Duffing oscillator, such as the modified perturbation methods [2,3], improved harmonic balance methods [4], energy balance method $[5,6]$, the frequency-amplitude formulation $[7,8]$. Meanwhile, the homotopy analysis method (HAM) [9] proposed by Liao has been proved to be one of the efficient analytical techniques in solving a variety of nonlinear Duffing problems. By the HAM, Hoseini et al. [10] study free vibrations of tapered beams and give an accurate analytical solution for the thirdorder Duffing equations; Qian et al. [11] obtain accurate analytical solutions for the fifth-order Duffing equations by considering vibrations of a restrained cantilever beam. For the seventh-order Duffing equations Qian et al. [12] get accurate analytical solutions by researching vibrations of an electrostatically actuated microbeam.

Thus, the prime objective of this paper is to explore the utility of the HAM for the generalized Duffing equa-

"Corresponding author. tion. All odd-type analytical results can be then involved in the generalized solution. In what follows, Section 2 presents natural frequency of the system obtained as a function of the initial amplitude and the general solution for any arbitrary odd power of $n$. In addition, the optimal HAM approach used to accelerate the convergence of solutions is also provided and discussed. In Section 3 two numerical examples are presented to examine the accuracy and validity of the proposed technique. In Section 4 the numerical results of the HAM are presented and compared with the numerical integration solutions. Finally, a conclusion summarizes the research findings in Section 5 .

\section{Solution Methodology}

In this section, we apply the HAM to solve the following nonlinear Duffing oscillator:

$$
\ddot{u}+u+a_{3} u^{3}+a_{5} u^{5}+a_{7} u^{7}+\cdots+a_{n} u^{n}=0,
$$

where $u$ is displacement and $a_{3}, a_{5}, a_{7}, \cdots, a_{n}$ ( $n$ is the odd number) are arbitrary constants. Subject to the following initial conditions:

$$
u(0)=A, \dot{u}(0)=0,
$$

where $A$ is an arbitrary constant. Under the transformation $\tau=\omega t$, Equations (1) and (2) can be rewritten as follows: 


$$
\begin{gathered}
\omega^{2} \ddot{u}+u+a_{3} u^{3}+a_{5} u^{5}+a_{7} u^{7}+\cdots+a_{n} u^{n}=0, \\
u(0)=A, \dot{u}(0)=0,
\end{gathered}
$$

where a dot $(\cdot)$ denotes differentiation with respect to $\tau$, and $\omega$ is the nonlinear frequency.

It is known that free oscillation of a conservative system without damping is a periodic motion and a harmonic function is the simplest type of periodic motion. So it can be expressed by the following base functions:

$$
\{\cos (2 m-1) \tau \mid m=1,2,3, \cdots\} .
$$

Taking into consideration the initial conditions in Equation (4), we choose the initial guess of $u(\tau)$ for the zeroth-order deformation equation as follows:

$$
u_{0}(\tau)=A \cos \tau .
$$

Thus, the auxiliary linear operator of a conservative system can be selected as

$$
L[u(\tau ; q)]=\omega_{0}^{2}\left(\frac{\partial^{2} u(\tau ; q)}{\partial \tau^{2}}+u(\tau ; q)\right) .
$$

The auxiliary linear operator $L$ is chosen in such a way that all solutions of the corresponding high-order formation equations exist and can be expressed by the general form of the base function. According to Equation (4), the nonlinear operator is written as:

$$
\begin{aligned}
& N[u(\tau ; q), \omega(q)] \\
& =[\omega(q)]^{2} \frac{\partial^{2} u(\tau ; q)}{\partial \tau^{2}}+u(\tau ; q) \\
& +\alpha_{3}[u(\tau ; q)]^{3}+\alpha_{5}[u(\tau ; q)]^{5} \\
& +\alpha_{7}[u(\tau ; q)]^{7}+\cdots+\alpha_{n}[u(\tau ; q)]^{n} .
\end{aligned}
$$

Then considering the homotopy function, we obtain the zeroth-order deformation equation as:

$$
(1-q) L\left[u(\tau ; q)-u_{0}(\tau)\right]=q \hbar N[u(\tau ; q), \omega(q)],
$$

where $q \in[0,1]$ and $\hbar$ are, respectively, embedding and convergence-control parameters. As $q$ changes from 0 to $1, u(\tau ; q)$ varies from the initial guess $u_{0}(\tau)$ to the unknown solution $u(\tau)$. Similarly, $\omega(q)$ varies from the initial guess frequency $\omega_{0}$ to the physical frequency $\omega$.

Then we make use of the Taylor series expansion to get

$$
\begin{gathered}
u(\tau ; q)=u_{0}(\tau)+\sum_{m=1}^{+\infty} u_{m}(\tau) q^{m}, \\
\omega(q)=\omega_{0}+\sum_{m=1}^{+\infty} \omega_{m} q^{m},
\end{gathered}
$$

in which

$$
\begin{gathered}
u_{m}(\tau)=\left.\frac{1}{m !} \frac{\partial^{m} u(\tau ; q)}{\partial q^{m}}\right|_{q=0}, \\
\omega_{m}=\left.\frac{1}{m !} \frac{\partial^{m} \omega(q)}{\partial q^{m}}\right|_{q=0},
\end{gathered}
$$

It is known that if $\hbar$ is properly chosen, the power series solutions in Equations (10) and (11) can be converged at $q=1$. So Equations (10) and (11) then become

$$
\begin{gathered}
u(\tau)=u_{0}(\tau)+\sum_{m=1}^{+\infty} u_{m}(\tau), \\
\omega=\omega_{0}+\sum_{m=1}^{+\infty} \omega_{m} .
\end{gathered}
$$

For the sake of simplicity, we define the following vectors:

$$
\begin{aligned}
& \boldsymbol{u}_{n}=\left\{u_{1}(\tau), u_{2}(\tau), \cdots, u_{n}(\tau)\right\}, \\
& \boldsymbol{\omega}_{n}=\left\{\omega_{0}, \omega_{1}, \cdots, \omega_{n}\right\} .
\end{aligned}
$$

By differentiating the zeroth-order deformation equation (9) $m$ times with respect to $q$, then the resulting equation is divided $m$ ! and setting $q=0$, it can be found the $m$ th-order deformation equation

$$
L\left[u_{m}(\tau)-\chi_{m} u_{m-1}(\tau)\right]=\hbar R_{m}\left(\boldsymbol{u}_{m-1} ; \boldsymbol{\omega}_{m-1}\right),
$$

with the initial conditions:

$$
u_{m}(0)=0, u_{m}^{\prime}(0)=0(m \geq 1),
$$

in which

$$
\begin{gathered}
\chi_{m}=\left\{\begin{array}{ll}
0, & \text { if } m \leq 1 \\
1, & \text { if } m>1
\end{array},\right. \\
R_{m}\left(\boldsymbol{u}_{m-1}, \boldsymbol{\omega}_{m-1}\right)=\left.\frac{1}{(m-1) !} \frac{\partial^{m-1} N[u(\tau ; q) ; \omega(q)]}{\partial q^{m-1}}\right|_{q=0} .
\end{gathered}
$$

Because odd nonlinearity of considered conservative system, $R_{m}$ can also be written as:

$$
R_{m}\left(\boldsymbol{u}_{m-1} ; \boldsymbol{\omega}_{m-1}\right)=\sum_{k=1}^{\phi(m)} d_{k}\left(\omega_{m-1}\right) \cos (2 k-1) \tau,
$$

where $\varphi(m)$ is an integer that depends on $m$.

Following the rule of solution expression and the linear operator $L$, the terms of $\cos \tau$ should not exist in $R_{m}$ of Equation (17), otherwise the so-called secular terms such as $\tau \cos \tau$ will appear in the final solutions. Therefore, their coefficients are set to zero as follows:

$$
\frac{1}{\pi} \int_{0}^{2 \pi}\left[\hbar R_{m}\left(\boldsymbol{u}_{m-1} ; \boldsymbol{\omega}_{m-1}\right)\right] \cos \tau \mathrm{d} \tau=0,
$$


The solutions of $\omega_{m-1}(m=1,2, \cdots)$ in Equations (17) and (22) can be determined successively. For the given values of $\alpha_{i}(i=3,5, \cdots, n)$ and $A$, we have the periodic solutions by the abovementioned analytical approach,

$$
\begin{aligned}
u_{m}(\tau)= & \chi_{m} u_{m-1}(\tau)+\frac{\hbar}{\omega_{0}^{2}} \sum_{k=2}^{\phi(m)} \frac{d_{k}\left(\omega_{m-1}\right) \cos (2 k-1) \tau}{\left(1-(2 k-1)^{2}\right)} \\
& +C_{1} \cos \tau+C_{0},
\end{aligned}
$$

where $C_{0}$ and $C_{1}$ can be determined by using the initial condition given in Equation (18).

Hence, the $m$ th-order approximations are

$$
u(\tau)=\sum_{i=0}^{m} u_{i}(\tau), \tau=\omega t
$$

in which

$$
\omega=\sum_{i=0}^{m} \omega_{i} .
$$

We know there are many optimal HAM approaches, which can be able to achieve faster convergent homotopy-series solutions $[13,14]$. In theory, we can define the exact residual error of the $m$ th-order of approximation as

$$
\Delta_{m}=\left|\sum_{i=0}^{m} \omega_{i}-\omega_{e x}\right| .
$$

where $\omega_{e x}$ is the exact nonlinear frequency of Equation (1) derived by using the numerical integration technique. It can be found $\Delta_{m}$ embraces the unknown convergencecontrol parameter $\hbar$. As $\Delta_{m}$ decreases more rapidly to zero, the speed of the convergence for the corresponding homotopy-series solution is faster [14]. The corresponding value of the convergence-control parameter $\hbar$ at the given order of approximation $m$ can be optimized and selected by minimizing the residual error $\Delta_{m}$.

\section{Numerical Results}

It can be obviously found the nonlinear Duffing oscillator for $n=3$ has a simple form. So in this section, we research the nonlinear Duffing oscillator for $n=5$ and $n=7$.

Firstly the nonlinear Duffing oscillator for $n=5$ has the following form:

$$
\ddot{u}+u+a_{3} u^{3}+a_{5} u^{5}=0 .
$$

The problem is solved and the general solution can be obtained based on the HAM in Section 2.

For $m=1$, one obtains $\omega_{0}$ from Equation (22) as follows

$$
\omega_{0}=\sqrt{1+\frac{3}{4} a_{3} A^{2}+\frac{5}{8} a_{5} A^{4}} .
$$

Making use of Equation (23) leads to

$$
u_{1}(\tau)=\left(\frac{b_{1,3} \hbar}{8 \omega_{0}^{2}}+\frac{b_{1,5} \hbar}{24 \omega_{0}^{2}}\right) \cos \tau-\frac{b_{1,3} \hbar}{8 \omega_{0}^{2}} \cos 3 \tau-\frac{b_{1,5} \hbar}{24 \omega_{0}^{2}} \cos 5 \tau .
$$

where

$$
\begin{gathered}
b_{1,3}=\frac{1}{4} A^{3} a_{3}+\frac{5}{16} A^{5} a_{5}, \\
b_{1,5}=\frac{1}{16} A^{5} a^{5} .
\end{gathered}
$$

For $m=2$, we substitute the solutions of $u_{0}(\tau)$, $u_{1}(\tau)$ and $\omega_{0}$ into Equation (22) to yield

$$
\begin{aligned}
\omega_{1}= & \frac{\hbar}{768 A \omega_{0}^{3}}\left(b_{1,3}\left(48+72 A^{2} a_{3}+75 A^{4} a_{5}-48 \omega_{0}^{2}\right)\right. \\
& \left.+b_{1,5}\left(16+36 A^{2} a_{3}+45 A^{4} a_{5}-16 \omega_{0}^{2}\right)\right) .
\end{aligned}
$$

From Equation (21), $R_{2}\left(\boldsymbol{u}_{1}, \boldsymbol{\omega}_{1}\right)$ are derived in the following:

$$
\begin{aligned}
R_{2}\left(\boldsymbol{u}_{1}, \boldsymbol{\omega}_{1}\right)= & b_{2,3} \cos 3 \tau+b_{2,5} \cos 5 \tau+b_{2,7} \cos 7 \tau \\
& +b_{2,9} \cos 9 \tau,
\end{aligned}
$$

where the coefficients $b_{2, i}(i=3,5,7,9)$ can be readily derived using Equation (21). In addition, $u_{2}(\tau)$ is given by

$$
\begin{aligned}
u_{2}(\tau)= & u_{1}(\tau)+\left(\frac{b_{2,3} \hbar}{8 \omega_{0}^{2}}+\frac{b_{2,5} \hbar}{24 \omega_{0}^{2}}+\frac{b_{2,7} \hbar}{48 \omega_{0}^{2}}+\frac{b_{2,9} \hbar}{80 \omega_{0}^{2}}\right) \cos \tau \\
& -\frac{b_{2,3} \hbar}{8 \omega_{0}^{2}} \cos 3 \tau-\frac{b_{2,5} \hbar}{24 \omega_{0}^{2}} \cos 5 \tau-\frac{b_{2,7} \hbar}{48 \omega_{0}^{2}} \cos 7 \tau \\
& -\frac{b_{2,9} \hbar}{80 \omega_{0}^{2}} \cos 9 \tau .
\end{aligned}
$$

For $m=3$, we can yield

$$
\begin{aligned}
\omega_{2}= & \frac{1}{23040 A \omega_{0}^{5}}\left(25 A^{3} a_{5}\left(27 b_{1,3}^{2}+24 b_{1,3} b_{1,5}+7 b_{1,5}^{2}\right) \hbar^{2}+150 A^{4} a_{5}\left(15 b_{1,3}+9 b_{1,5}+15 b_{2,3}+9 b_{2,5}+5 b_{2,7}+3 b_{2,9}\right) \hbar \omega_{0}^{2}\right. \\
& +108 A^{2} a_{3}\left(20 b_{1,3}+10 b_{1,5}+20 b_{2,3}+10 b_{2,5}+5 b_{2,7}+3 b_{2,9}\right) \hbar \omega_{0}^{2}+15 A\left(a_{3}\left(27 b_{1,3}^{2}+18 b_{1,3} b_{1,5}+5 b_{1,5}^{2}\right) \hbar^{2}-768 \omega_{0}^{4} \omega_{1}^{2}\right) \\
& \left.-48 \hbar \omega_{0}^{2}\left(\left(30 b_{2,3}+10 b_{2,5}+5 b_{2,7}+3 b_{2,9}\right)\left(-1+\omega_{0}^{2}\right)+30 b_{1,3}\left(-1+\omega_{0}^{2}+2 \omega_{0} \omega_{1}\right)+10 b_{1,5}\left(-1+\omega_{0}^{2}+2 \omega_{0} \omega_{1}\right)\right)\right) \\
& R_{3}\left(\boldsymbol{u}_{2}, \boldsymbol{\omega}_{2}\right)=b_{3,3} \cos 3 \tau+b_{3,5} \cos 5 \tau+b_{3,7} \cos 7 \tau+b_{3,9} \cos 9 \tau+b_{3,11} \cos 11 \tau+b_{3,13} \cos 13 \tau,
\end{aligned}
$$




$$
\begin{aligned}
u_{3}(\tau)= & u_{2}(\tau)+\left(\frac{b_{3,3} \hbar}{8 \omega_{0}^{2}}+\frac{b_{3,5} \hbar}{24 \omega_{0}^{2}}+\frac{b_{3,7} \hbar}{48 \omega_{0}^{2}}+\frac{b_{3,9} \hbar}{80 \omega_{0}^{2}}+\frac{b_{3,11} \hbar}{120 \omega_{0}^{2}}+\frac{b_{3,13} \hbar}{168 \omega_{0}^{2}}+\frac{b_{3,19} \hbar}{360 \omega_{0}^{2}}\right) \cos \tau-\frac{b_{3,3} \hbar}{8 \omega_{0}^{2}} \cos 3 \tau \\
& -\frac{b_{3,5} \hbar}{24 \omega_{0}^{2}} \cos 5 \tau-\frac{b_{3,7} \hbar}{48 \omega_{0}^{2}} \cos 7 \tau-\frac{b_{3,9} \hbar}{80 \omega_{0}^{2}} \cos 9 \tau-\frac{b_{3,11} \hbar}{120 \omega_{0}^{2}} \cos 11 \tau-\frac{b_{3,13} \hbar}{168 \omega_{0}^{2}} \cos 13 \tau,
\end{aligned}
$$

where all coefficients $b_{3, i}(i=3,5, \cdots, 13)$ can be computed from Equation (21).

According to Equations (24) and (25), the corresponding third-order analytical approximation for Equation (27) is

$$
u(\tau)=u_{0}(\tau)+u_{1}(\tau)+u_{2}(\tau)+u_{3}(\tau), \tau=\omega t,
$$

where

$$
\omega=\omega_{0}+\omega_{1}+\omega_{2} .
$$

The higher-order approximations for $\omega$ and $u(\tau)$ can be derived in a similar manner.

Secondly we consider the nonlinear Duffing oscillator for $n=7$ :

$$
\ddot{u}+u+a_{3} u^{3}+a_{5} u^{5}+a_{7} u^{7}=0,
$$

We can get some approximations for $\omega$ and $u(\tau)$ according to Section 2 in a similar manner.

For $m=1$, one obtains

$$
\begin{gathered}
\omega_{0}=\sqrt{1+\frac{3}{4} a_{3} A^{2}+\frac{5}{8} a_{5} A^{4}+\frac{35}{64} a_{7} A^{6}}, \\
u_{1}(\tau)=\left(\frac{b_{1,3} \hbar}{8 \omega_{0}^{2}}+\frac{b_{1,5} \hbar}{24 \omega_{0}^{2}}+\frac{b_{1,7} \hbar}{48 \omega_{0}^{2}}\right) \cos \tau \\
-\frac{b_{1,3} \hbar}{8 \omega_{0}^{2}} \cos 3 \tau-\frac{b_{1,5} \hbar}{24 \omega_{0}^{2}} \cos 5 \tau-\frac{b_{1,7} \hbar}{48 \omega_{0}^{2}} \cos 7 \tau,
\end{gathered}
$$

where

$$
\begin{aligned}
& b_{1,3}=\frac{1}{4} A^{3} a_{3}+\frac{5}{16} A^{5} a_{5}+\frac{21}{64} A^{7} a_{7}, \\
& b_{1,5}=\frac{1}{4} A^{5} a_{5}+\frac{7}{64} A^{7} a_{7}, \\
& b_{1,7}=\frac{1}{64} A^{7} a^{7} .
\end{aligned}
$$

For $m=2$, we can yield

$$
\begin{aligned}
& \omega_{1}=\frac{\hbar}{3072 A \omega_{0}^{3}}\left(6 b_{1,3}\left(32+48 A^{2} a_{3}+50 A^{4} a_{5}+49 A^{6} a_{7}-32 \omega_{0}^{2}\right)+b_{1,7}\left(32+72 A^{2} a_{3}+100 A^{4} a_{5}+119 A^{6} a_{7}-32 \omega_{0}^{2}\right)\right. \\
& \left.\quad+4 b_{1,5}\left(16+36 A^{2} a_{3}+45 A^{4} a_{5}+49 A^{6} a_{7}-16 \omega_{0}^{2}\right)\right), \\
& R_{2}\left(\boldsymbol{u}_{1}, \boldsymbol{\omega}_{1}\right)=b_{2,3} \cos 3 \tau+b_{2,5} \cos 5 \tau+b_{2,7} \cos 7 \tau+b_{2,9} \cos 9 \tau+b_{2,11} \cos 11 \tau+b_{2,13} \cos 13 \tau, \\
& u_{2}(\tau)=u_{1}(\tau)+\left(\frac{b_{2,3} \hbar}{8 \omega_{0}^{2}}+\frac{b_{2,5} \hbar}{24 \omega_{0}^{2}}+\frac{b_{2,7} \hbar}{48 \omega_{0}^{2}}+\frac{b_{2,9} \hbar}{80 \omega_{0}^{2}}+\frac{b_{2,11} \hbar}{120 \omega_{0}^{2}}+\frac{b_{2,13} \hbar}{168 \omega_{0}^{2}}\right) \cos \tau-\frac{b_{2,3} \hbar}{8 \omega_{0}^{2}} \cos 3 \tau-\frac{b_{2,5} \hbar}{24 \omega_{0}^{2}} \cos 5 \tau \\
& \quad-\frac{b_{2,7} \hbar}{48 \omega_{0}^{2}} \cos 7 \tau-\frac{b_{2,9} \hbar}{80 \omega_{0}^{2}} \cos 9 \tau-\frac{b_{2,11} \hbar}{120 \omega_{0}^{2}} \cos 11 \tau-\frac{b_{2,13} \hbar}{168 \omega_{0}^{2}} \cos 13 \tau .
\end{aligned}
$$

For $m=3$, one arrives at

$$
\begin{aligned}
\omega_{2}= & \frac{1}{10321920 A \omega_{0}^{5}}\left(735 A^{5} a_{7}\left(504 b_{1,3}^{2}+528 b_{1,3} b_{1,5}+164 b_{1,5}^{2}+228 b_{1,3} b_{1,7}+168 b_{1,5} b_{1,7}+53 b_{1,7}^{2}\right) \hbar^{2}\right. \\
& +5600 A^{3} a_{5}\left(54 b_{1,3}^{2}+14 b_{1,5}^{2}+13 b_{1,5} b_{1,7}+4 b_{1,7}^{2}+6 b_{1,3}\left(8 b_{1,5}+3 b_{1,7}\right)\right) \hbar^{2} \\
& +11760 A^{6} a_{7}\left(84 b_{1,3}+56 b_{1,5}+34 b_{1,7}+14 b_{2,11}+10 b_{2,13}+84 b_{2,3}+56 b_{2,5}+34 b_{2,7}+21 b_{2,9}\right) \hbar \omega_{0}^{2} \\
& +9600 A^{4} a_{5}\left(105 b_{1,3}+63 b_{1,5}+35 b_{1,7}+14 b_{2,11}+10 b_{2,13}+105 b_{2,3}+63 b_{2,5}+35 b_{2,7}+21 b_{2,9}\right) \hbar \omega_{0}^{2} \\
& +6912 A^{2} a_{3}\left(140 b_{1,3}+70 b_{1,5}+35 b_{1,7}+14 b_{2,11}+10 b_{2,13}+140 b_{2,3}+70 b_{2,5}+35 b_{2,7}+216 b_{2,9}\right) \hbar \omega_{0}^{2} \\
& +1680 A\left(a_{3}\left(108 b_{1,3}^{2}+20 b_{1,5}^{2}+16 b_{1,5} b_{1,7}+5 b_{1,7}^{2}+24 b_{1,3}\left(3 b_{1,5}+b_{1,7}\right)\right) \hbar^{2}-3072 \omega_{0}^{4} \omega_{1}^{2}\right) \\
& -3072 \hbar \omega_{0}^{2}\left(-35 b_{1,7}-14 b_{2,11}-10 b_{2,13}-210 b_{2,3}-70 b_{2,5}-35 b_{2,7}-21 b_{2,9}+35 b_{1,7} \omega_{0}^{2}+14 b_{2,11} \omega_{0}^{2}\right. \\
& +10 b_{2,13} \omega_{0}^{2}+210 b_{2,3} \omega_{0}^{2}+70 b_{2,5} \omega_{0}^{2}+35 b_{2,7} \omega_{0}^{2}+21 b_{2,9} \omega_{0}^{2}+70 b_{1,7} \omega_{0} \omega_{1} \\
& \left.\left.+210 b_{1,3}\left(-1+\omega_{0}^{2}+2 \omega_{0} \omega_{1}\right)+70 b_{1,5}\left(-1+\omega_{0}^{2}+2 \omega_{0} \omega_{1}\right)\right)\right),
\end{aligned}
$$




$$
\begin{aligned}
R_{3}\left(\boldsymbol{u}_{2}, \boldsymbol{\omega}_{2}\right)= & b_{3,3} \cos 3 \tau+b_{3,5} \cos 5 \tau+b_{3,7} \cos 7 \tau+b_{3,9} \cos 9 \tau+b_{3,11} \cos 11 \tau+b_{3,13} \cos 13 \tau \\
& +b_{3,15} \cos 15 \tau+b_{3,17} \cos 17 \tau+b_{3,19} \cos 19 \tau \\
u_{3}(\tau)= & \left.\frac{b_{3,3} \hbar}{8 \omega_{0}^{2}}+\frac{b_{3,5} \hbar}{24 \omega_{0}^{2}}+\frac{b_{3,7} \hbar}{48 \omega_{0}^{2}}+\frac{b_{3,9} \hbar}{80 \omega_{0}^{2}}+\frac{b_{3,11} \hbar}{120 \omega_{0}^{2}}+\frac{b_{3,13} \hbar}{168 \omega_{0}^{2}}+\frac{b_{3,15} \hbar}{224 \omega_{0}^{2}}+\frac{b_{3,17} \hbar}{288 \omega_{0}^{2}}+\frac{b_{3,19} \hbar}{360 \omega_{0}^{2}}\right) \cos \tau \\
& -\frac{b_{3,3} \hbar}{8 \omega_{0}^{2}} \cos 3 \tau-\frac{b_{3,5} \hbar}{24 \omega_{0}^{2}} \cos 5 \tau-\frac{b_{3,7} \hbar}{48 \omega_{0}^{2}} \cos 7 \tau-\frac{b_{3,9} \hbar}{80 \omega_{0}^{2}} \cos 9 \tau-\frac{b_{3,11} \hbar}{120 \omega_{0}^{2}} \cos 11 \tau-\frac{b_{3,13} \hbar}{168 \omega_{0}^{2}} \cos 13 \tau \\
& -\frac{b_{3,15} \hbar}{224 \omega_{0}^{2}} \cos 15 \tau-\frac{b_{3,17} \hbar}{288 \omega_{0}^{2}} \cos 17 \tau-\frac{b_{3,19} \hbar}{360 \omega_{0}^{2}} \cos 19 \tau+u_{2}(\tau)
\end{aligned}
$$

where all coefficients $b_{2, i}(i=3,5, \cdots, 13)$ and $b_{3, i}(i=3,5, \cdots, 19)$ can be computed from Equation (21).

Similarly, the corresponding third-order analytical approximation for Equation (40) is

$$
u(\tau)=u_{0}(\tau)+u_{1}(\tau)+u_{2}(\tau)+u_{3}(\tau), \tau=\omega t
$$

where

$$
\omega=\omega_{0}+\omega_{1}+\omega_{2} .
$$

The higher-order approximations for $\omega$ and $u(\tau)$ can be derived in a similar manner.

\section{Numerical Discussion}

In order to demonstrate the effectiveness of the presenting method, the asymptotic analytical solutions obtained by the HAM are directly compared to the published analytical and numerical integration solutions. Tables 1-4 present the results of He's frequency-amplitude formulation $\omega_{F A F}[15]$, He's energy balance method $\omega_{E B M}$ [15], presents approach $\omega_{\text {HAM }}$ and exact solution $\omega_{e x}$ for various parameters $a_{i}$ and amplitudes $A$.

In comparison with the exact solutions $\omega_{e x}$ in Tables 1 and 2, it can be distinctly observed the second-order HAM solutions $\omega_{\text {HAM }}$ for $\hbar=-1$ are better than the first-order solutions of $\omega_{F A F}$ and $\omega_{E B M}$. Moreover it can be checked that the HAM has a better performance for large amplitudes of motion.

In order to illustrate the effect of the convergencecontrol parameter $\hbar$, we can make use of Equation (26) to optimize $\hbar$ and draw the $\Delta_{m} \square \hbar$ curve to obtain the optimal convergence-control parameter $\hbar$.

By Figure 1, we get the optimal convergence-control parameters $\hbar$ in Table 3 for Modes 1-6. In Table 3 and Table 4, the results of $\omega_{\text {HAM }}$ for the previous cases are refined in using the optimal parameters $\hbar$. Because the parameter $\hbar=-1$ is almost equal to the optimal value $\hbar$ in the Tables, thus, it is not very evident to show the power about the auxiliary parameter $\hbar$. But the value of $\hbar$ is one of the dominant factors in the HAM to extend its validity and flexibility. This also shows the good performance of investigating the nonlinear Duffing equations in this paper using the HAM.

Figures 2 and $\mathbf{3}$ show the phase portrait diagrams and time history responses of Tables 1 and 2 where $\hbar=-1$ is chosen. We can clearly find that the third-order HAM solutions are in good agreement with the numerical integration solutions even if the amplitude is larger.

\section{Conclusion}

In this study, the HAM has intensively studied the generalized Duffing equation. The general frequencies and periodic solutions are presented for any arbitrary oddtype of nonlinearity. The purposes of this paper are not only to formulate the asymptotic approximate solutions for the nonlinear Duffing oscillators, but also to furnish a guidance to establish the higher-order asymptotic analytical approximations if necessary. Moreover, it is found that the accuracy of the HAM is affected by the selection

Table 1. Comparison of the exact and approximate frequencies corresponding to various parameters in Equation (27) for $\hbar=-1$.

\begin{tabular}{cccccccc}
\hline$A$ & $a_{3}$ & $a_{5}$ & $\omega_{\text {FAF }}$ & $\omega_{\text {ЕВM }}$ & $\omega_{\text {НAM }}$ & $\omega_{\text {ex }}$ \\
\hline 1 & 1 & 1 & 1.541 & 1.5275 & 1.5238 & 1.5235 \\
0.5 & 10 & 10 & 1.807 & 1.7998 & 1.7851 & 1.7847 \\
0.1 & 100 & 100 & 1.325 & 1.3250 & 1.3200 & 1.3200 \\
5 & 0.1 & 0.1 & 6.475 & 6.2716 & 6.1699 & 6.1473 \\
10 & 0.05 & 0.05 & 17.81 & 17.216 & 16.913 & 16.841 \\
20 & 0.01 & 0.01 & 31.6 & 30.615 & 30.070 & 29.940 \\
\hline
\end{tabular}


Table 2. Comparison of the exact and approximate frequencies corresponding to various parameters in Equation (40) for $\hbar=-1$.

\begin{tabular}{ccccccccc}
\hline$A$ & $a_{3}$ & $a_{5}$ & $a_{7}$ & $\omega_{\text {FAF }}$ & $\omega_{\text {ЕBM }}$ & $\omega_{\text {HAM }}$ & $\omega_{\text {ex }}$ \\
\hline 1 & 1 & 1 & 1 & 1.7093 & 1.6739 & 1.6763 & 1.6753 \\
0.5 & 10 & 10 & 10 & 1.8305 & 1.8201 & 1.8064 & 1.8060 \\
0.1 & 100 & 100 & 100 & 1.3252 & 1.3251 & 1.3200 & 27.692 & 27.386 \\
5 & 0.1 & 0.1 & 0.1 & 29.940 & 27.780 & 1.3200 & 153.63 & 151.87 \\
10 & 0.05 & 0.05 & 0.05 & 166.31 & 154.05 & 548.57 & 547.14 & 540.81 \\
\hline
\end{tabular}

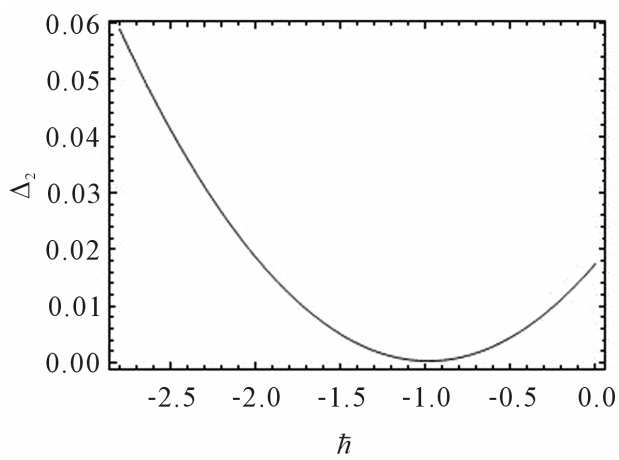

Mode 1

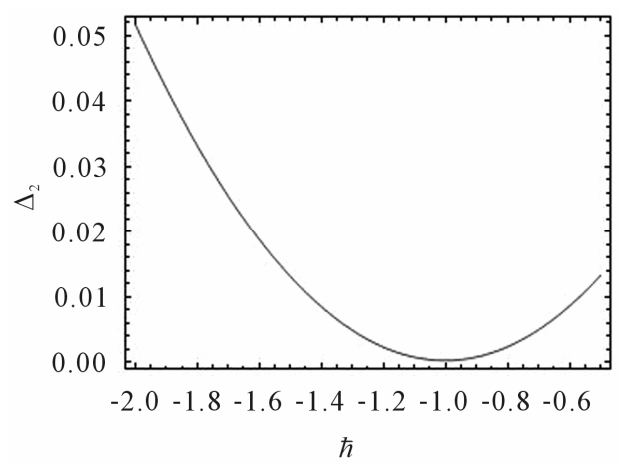

Mode 3

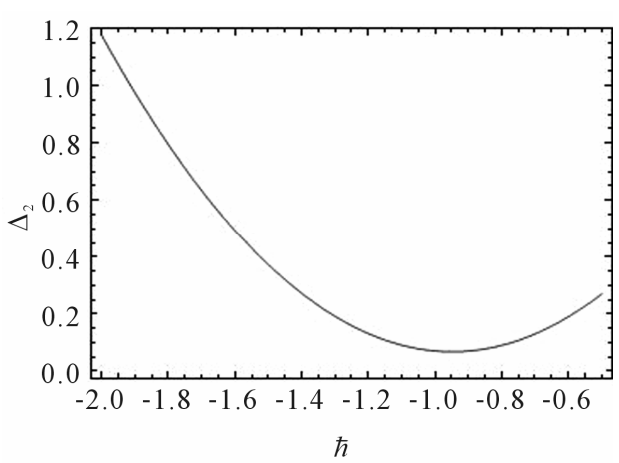

Mode 5

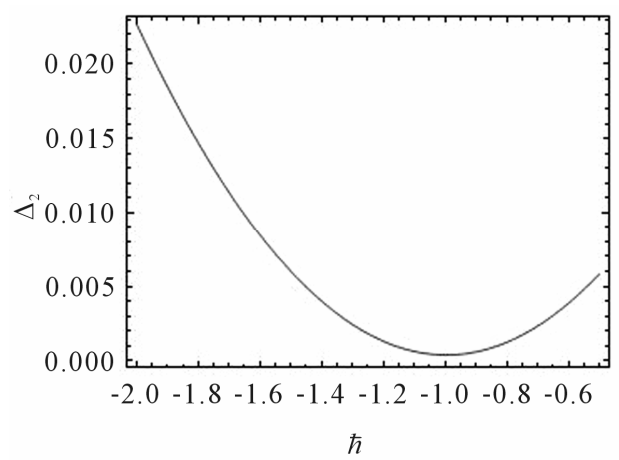

Mode 2

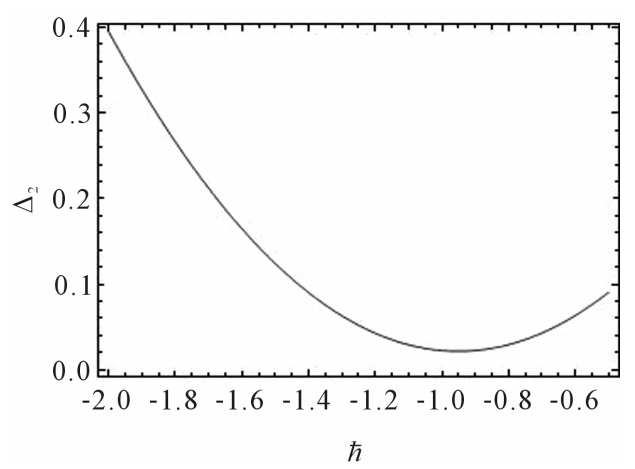

Mode 4

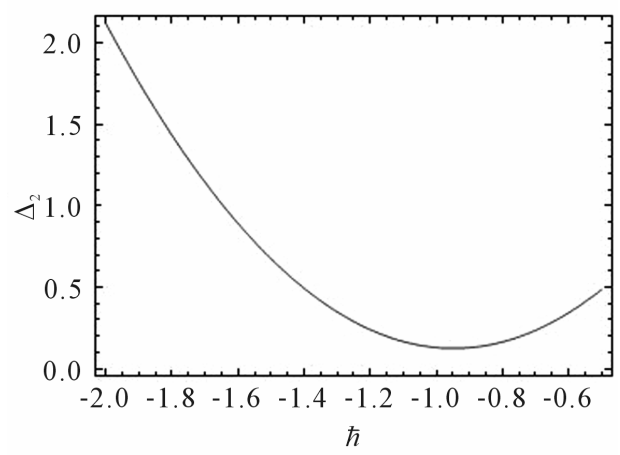

Mode 6

Figure 1. The selection of optimal convergence-control parameters $\hbar$ in Table 3 for Modes 1 - 6 . 


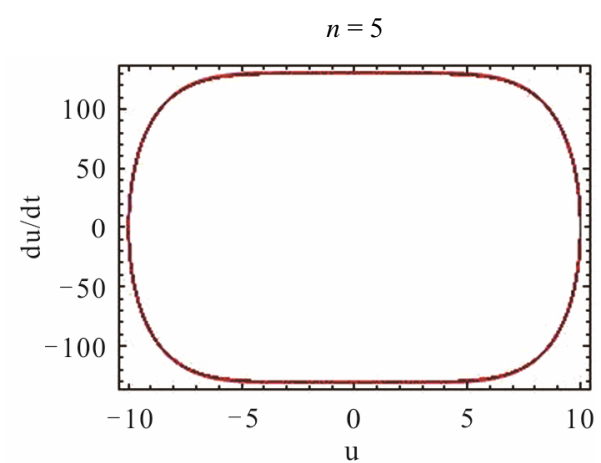

(a)

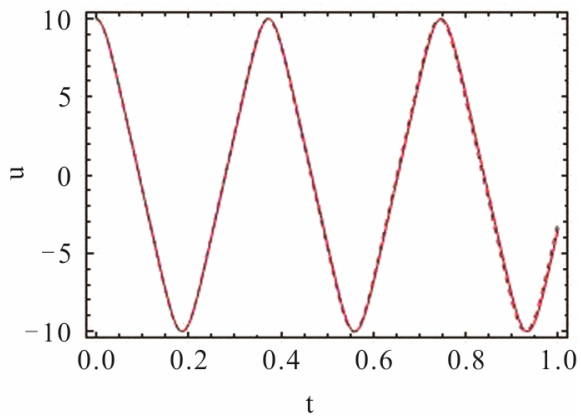

(b)

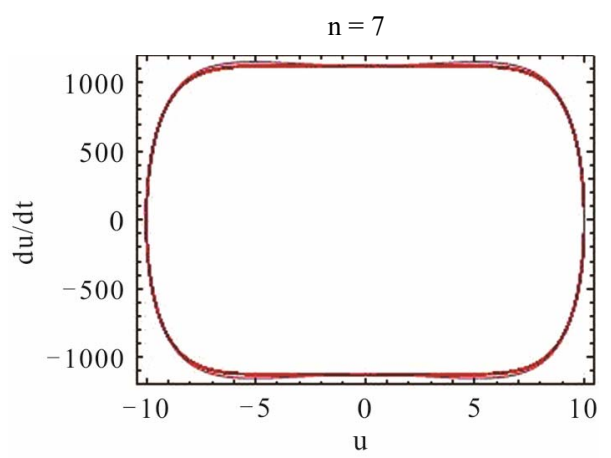

(a)

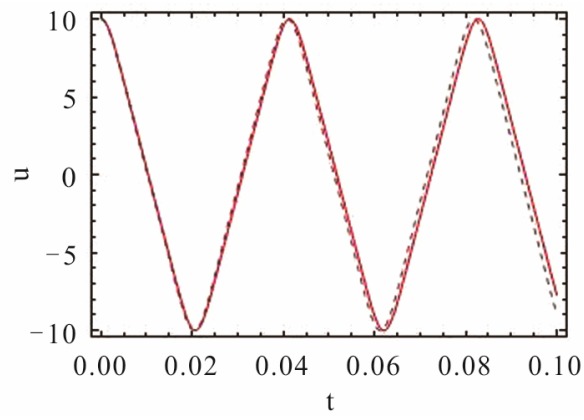

(b)

Figure 2. Comparison of the approximate and numerical integration solutions for $A=10$. (a) Phase portrait diagram; (b) Time history response.



(a)

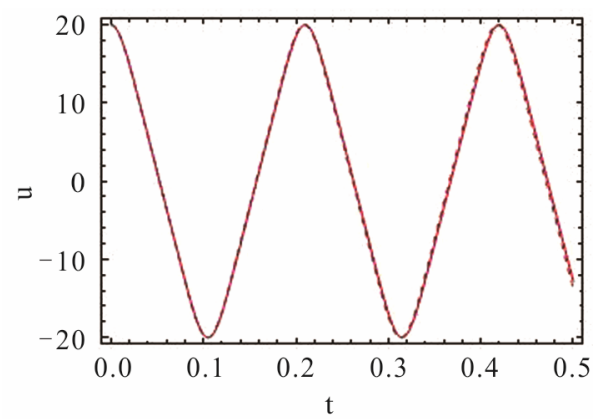

(b)

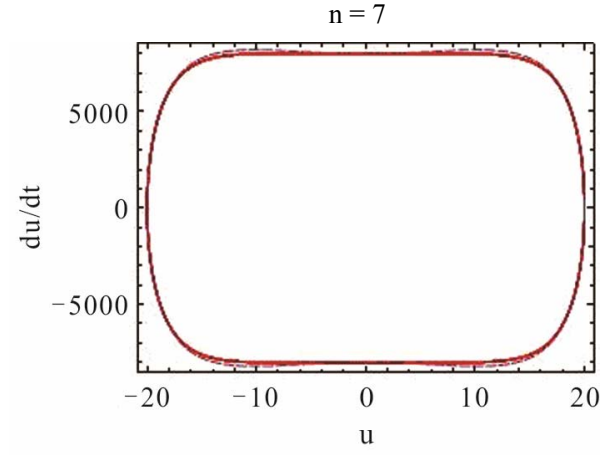

(a)



(b)

HAM approximate solution

Numerical integration solution

Figure 3. Comparison of the approximate and numerical integration solutions for $A=20$. (a) Phase portrait diagram; (b) Time history response. 
Table 3. Comparison of the approximate frequencies using the optimal convergence-control parameter $\hbar$ with the exact solution for $n=5$.

\begin{tabular}{cccccc}
\hline$A$ & $a_{3}$ & $a_{5}$ & $\hbar$ & $\omega_{\text {нам }}$ & $\omega_{\text {ех }}$ \\
\hline 1 & 1 & 1 & -0.9841 & 1.52386 & 1.52359 \\
0.5 & 10 & 10 & -0.9961 & 1.7851 & 1.78471 \\
0.1 & 100 & 100 & -1.0016 & 1.32006 & 1.32003 \\
5 & 0.1 & 0.1 & -0.9509 & 6.16916 & 6.14739 \\
10 & 0.05 & 0.05 & -0.9486 & 16.9103 & 16.8416 \\
20 & 0.01 & 0.01 & -0.9480 & 30.0657 & 29.9408 \\
\hline
\end{tabular}

Table 4. Comparison of the approximate frequencies using the optimal convergence-control parameter $\hbar$ with the exact solution for $n=7$.

\begin{tabular}{cccccccc}
\hline$A$ & $a_{3}$ & $a_{5}$ & $a_{7}$ & $\hbar$ & $\omega_{\text {нам }}$ & $\omega_{\text {ех }}$ \\
\hline 1 & 1 & 1 & 1 & -0.9604 & 1.67625 & 1.67534 \\
0.5 & 10 & 10 & 10 & -0.9924 & 1.80648 & 1.80602 \\
0.1 & 100 & 100 & 100 & -1.0016 & 1.32008 & 1.32005 \\
5 & 0.1 & 0.1 & 0.1 & -0.8848 & 27.6536 & 27.3865 \\
10 & 0.05 & 0.05 & 0.05 & -0.8826 & 153.407 & 151.874 \\
20 & 0.01 & 0.01 & 0.01 & -0.8821 & 546.318 & 540.817 \\
\hline
\end{tabular}

of appropriate convergence-control parameter $\hbar$. To further improve the accuracy of solutions, the parameter $\hbar$ can be optimized and determined by the minimization of the residual error $\Delta_{m}$ for any given order approximation $m$. Besides, illustrative examples are applied as crucial evidence to support that the HAM is effective for the quantitative analysis of nonlinear problems. Finally, this paper suggests to readers to apply the method for solving nonlinear large-amplitude oscillations because of its accuracy, reliability and simplicity.

\section{Acknowledgements}

The authors gratefully acknowledge the support of the National Natural Science Foundation of China (NNSFC) through grant No.11202189 and the National Natural Science Foundation of Zhejiang through grant No. LY12A02002.

\section{REFERENCES}

[1] I. Kovacic and M. J. Brennan, "The Duffing Equation: Nonlinear Oscillators and Their Behaviour," John Wiley \& Sons, Hoboken, 2011. doi:10.1002/9780470977859

[2] P. Amore and A. Aranda, "Improved Lindstedt-Poincaré Method for the Solution of Nonlinear Problems," Journal of Sound and Vibration, Vol. 283, No. 3-5, 2005, pp. 1115-1136. doi:10.1016/j.jsv.2004.06.009
[3] R. R. Pušenjak, "Extended Lindstedt-Poincare Method for Non-Stationary Resonances of Dynamical Systems with Cubic Nonlinearities," Journal of Sound and Vibration, Vol. 314, No. 1-2, 2008, pp. 194-216. doi:10.1016/i.jsv.2008.01.002

[4] B. S. Wu and P. S. Li, "A method for Obtaining Approximate Analytic Periods for a Class of Nonlinear Oscillators," Meccanica, Vol. 36, No. 2, 2001, pp. 167-176. doi:10.1023/A:1013067311749

[5] H. L. Zhang, "Periodic Solutions for Some Strongly Nonlinear Oscillations by He's Energy Balance Method," Computers and Mathematics with Applications, Vol. 58, No. 11-12, 2009, pp. 2480-2485. doi:10.1016/j.camwa.2009.03.068

[6] I. Mehdipour, D. D. Ganji and M. Mozaffari, "Application of the Energy Balance Method to Nonlinear Vibrating Equations," Current Applied Physics, Vol. 10, No. 1, 2010, pp. 104-112. doi:10.1016/j.cap.2009.05.016

[7] L. Geng and X. C. Cai, "He's Frequency Formulation for Nonlinear Oscillators," European Journal of Physics, Vol. 28, 2007, pp. 923-931. doi:10.1088/0143-0807/28/5/016

[8] J. Fan, "He's Frequency-Amplitude Formulation for the Duffing Harmonic Oscillator," Computers and Mathematics with Applications, Vol. 58, No. 11-12, 2009, pp. 2473-2476. doi:10.1016/j.camwa.2009.03.049

[9] S. J. Liao, "Beyond Perturbation: Introduction to the Homotopy Analysis Method," Chapman \& Hall, Boca Raton, 2003. doi:10.1201/9780203491164

[10] S. H. Hoseini, T. Pirbodaghi, M. T. Ahmadian and G. H. 
Farrahi, "On the Large Amplitude Free Vibrations of Tapered Beams: An Analytical Approach," Mechanics Research Communications, Vol. 36, No. 8, 2009, pp. 892897. doi:10.1016/i.mechrescom.2009.08.003

[11] Y. H. Qian, S. K. Lai, W. Zhang and Y. Xiang, "Study on Asymptotic Analytical Solutions Using HAM for Strongly Nonlinear Vibrations of a Restrained Cantilever Beam with an Intermediate Lumped Mass," Numerical Algorithms, Vol. 58, No. 3, 2011, pp. 293-314. doi:10.1007/s11075-011-9456-7

[12] Y. H. Qian, D. X. Ren, S. K. Lai and S. M. Chen, "Analytical Approximations to Nonlinear Vibration of an Electrostatically Actuated Microbeam," Communications in Nonlinear Science and Numerical Simulation, Vol. 17, No. 4, 2012, pp. 1947-1955. doi:10.1016/j.cnsns.2011.09.018

[13] R. A. Van Gorder and K. Vajravelu, "On the Selection of
Auxiliary Functions, Operators, and Convergence Control Parameters in the Application of the Homotopy Analysis Method to Nonlinear Differential Equations: A General Approach," Communication in Nonlinear Sciences and Numerical Simulation, Vol. 14, No. 12, 2009, pp. 40784089. doi:10.1016/j.cnsns.2009.03.008

[14] S. J. Liao, "An Optimal Homotopy-Analysis Approach for Strongly Nonlinear Differential Equations," Communication in Nonlinear Sciences and Numerical Simulation, Vol. 15, No. 8, 2010, pp. 2003-2016. doi:10.1016/j.cnsns.2009.09.002

[15] Y. Davood, A. Hassan, S. Zia and K. Mohammad, "Frequency Analysis of Strongly Nonlinear Generalized Duffing Oscillators Using He's Frequency-Amplitude Formulation and He's Energy Balance Method," Computers and Mathematics with Applications, Vol. 59, No. 9, 2010, pp. 3222-3228. doi:10.1016/j.camwa.2010.03.013 